\title{
How do patients feel about taking part in clinical trials in emergency care?
}

\author{
Joseph M Buckley, Andy D Irving, Steve Goodacre
}

\begin{abstract}
- Additional material is published online only. To view please visit the journal online (http://dx.doi.org/10.1136/ emermed-2015-205146).
\end{abstract}

School of Health and Related Research, University of Sheffield, Sheffield, South Yorkshire, UK

\section{Correspondence to} Joseph M Buckley, School of Health and Related Research, University of Sheffield, 30 Regent Street, Sheffield, South Yorkshire S1 4DA, UK; joebuckley@hotmail.co.uk

Prior presentations: Poster presentation of the results of the same study, College of Emergency Medicine Conference, 9 September 2014, Exeter University, Exeter UK

Received 19 June 2015 Revised 6 November 2015 Accepted 19 December 2015 Published Online First 12 January 2016

\section{SLinked}

- http://dx.doi.org/10.1136/ emermed-2016-205811

\section{CrossMark}

To cite: Buckley JM,

Irving $A D$, Goodacre $S$.

Emerg Med J 2016;33

376-380.

\section{ABSTRACT}

Background There is little in-depth research into how patients feel about emergency medical trials, and what influences these feelings.

Objectives To investigate patients' feelings on taking part in emergency medical research, particularly trials conducted without prospective consent.

Methods Seventeen inpatients, all recently admitted with a medical emergency, were interviewed. Questions focused on feelings on taking part in hypothetical trials, particularly trials conducted with deferred consent.

Results Five main themes were identified. Level of trust in the medical profession-high levels of trust tended to correlate with willingness to participate in trials. Previous bad healthcare experiences tended to diminish trust. Concerns for personal well-being — patients identified a conflict between aversion to unknown side effects and desire for access to newer and potentially better treatments. Some would be less inclined to participate in research if they were severely unwell, some more so. Altruism - many cited the importance of helping to advance medical knowledge and of 'giving back to the health service'. Concerns over autonomy — some felt that deferred consent was a violation of personal autonomy. Uncertainty - many patients seemed to struggle to understand the more complex concepts discussed.

Conclusions Patients are broadly trusting, and open to participating in emergency medical trials, but want to be kept as informed as possible throughout the process. Willingness may be improved by providing more complete explanations, although this may be limited by the complexity of relevant concepts. Good communication and improved public understanding of clinical trials would likely increase acceptance of emergency care research.

\section{INTRODUCTION AND BACKGROUND}

The need for clinical research in emergency medicine is well established. The increasing number of emergency medical trials carried out in recent years has led to improved clinical practice worldwide and significantly improved patient outcomes during emergency admissions. However, carrying out clinical trials in the emergency setting can be ethically problematic; the added element of time pressure and the distressed mental state of many patients both provide barriers to gaining prospective informed consent for any experimental intervention. In such situations, gaining informed consent may delay the administration of treatment for long enough to significantly diminish the treatment's effectiveness. ${ }^{1}$ This would be denying the patient the full benefit of a potentially beneficial treatment, and it would have the potential to affect the results

\section{Key messages}

What is already known on this subject

- Public understanding of the workings of clinical trials is rather patchy, particularly emergency trials that often involve deferred consent.

- Studies conducted in the USA suggest that the public are uneasy with the idea of such research taking place.

- Although there is little existing research, there is some evidence to suggest a slightly more permissive attitude towards such research in Europe.

\section{What might this study add?}

- This study found that the attitudes towards emergency medical research among those surveyed were largely positive, so long as every effort was made to keep the participant as informed as possible.

- It also found that levels of understanding and awareness of such research in the study sample were relatively low.

of the trial and therefore also any recommendations made.

As a result, measures are often taken to streamline the consent process. These include optional verbal consent rather than written consent (eg, the $3 \mathrm{Mg}^{2}$ and IST-3 trials ${ }^{3}$ ); using an abridged version of the patient information sheet prospectively and giving the patient the remaining details after administration of the treatment (eg, the $3 \mathrm{Mg}$ study); or even, in cases where the patient does not have capacity to consent, deferring consent entirely until after the treatment has been given and the patient has regained capacity (eg, the IST-3 and CRASH2 trials $\left.{ }^{4}\right)$.

Naturally, many of these measures present significant ethical challenges-the idea of deferred consent in particular seems to run counter to the principles of the Declaration of Helsinki. However, not carrying out such trials could also be seen as unethical, as it would deny the global community the life-saving advances in practice often brought about as a result of them. Given the complex ethical issues involved, there is a clear need to understand patient and public opinion on the principle and practicalities of all emergency medicine research. However, the universal nature of emergency care makes it difficult to sound out public opinion of trials in advance of their commencement, as there is no clear audience or group who 
think of themselves as the typical service users. In the USA, the Food and Drugs Administration (FDA) mandates that prior to the commencement of an emergency medicine trial, there must be 'consultation (including, where appropriate, consultation carried out by the IRB) with representatives of the communities in which the research will be conducted and from which the subjects will be drawn'. ${ }^{5}$ In the UK, there is no such similar specific legal mandate for consultation with the local public; however, ethical approval is of course still required, and evidence of input from patient and public involvement (PPI) groups at the design stage is expected before this can be granted.

In this study, we aimed to investigate in depth how members of the public felt about the ethical issues involved in such research.

A search of WebOfScience and ScienceDirect demonstrated a relative lack of research into this area. A selection of papers were found, dating from 2003 to 2014. The papers identified included qualitative, quantitative and mixed methods studies.

Several broad trends appeared across the papers. The first of these was that most members of the public surveyed were uneasy with the idea of enrolling patients in clinical trials without their prior consent. ${ }^{6-11}$ There was also a general trend across the studies of people with prior experience of clinical research (particularly in an emergency setting) being more willing to take part in hypothetical research of any kind in the future. ${ }^{9} 1112$ This suggests that one reason some people hesitate to take part in clinical trials may simply be a fear of the unknown (or a misunderstanding about what such participation might involve), which has been allayed in those who have done so before.

The studies showed that public understanding of clinical trials was variable-one paper in particular investigated the reasons why a selection of members of the public decided to preemptively opt out of an emergency medical trial, and found an alarming collection of misunderstandings about the process. ${ }^{8}$

Table 1 Summary of interviewee demographics

\begin{tabular}{ll}
\hline Type of participant & N (total $\mathbf{n}=\mathbf{1 7}$ ) \\
\hline Age & 2 \\
$18-24$ & 3 \\
$25-34$ & 3 \\
$35-44$ & 3 \\
$45-54$ & 2 \\
$55-64$ & 4 \\
$65-74$ & \\
Gender & 8 \\
Female & 9 \\
Male & \\
Ethnicity & 14 \\
White, British & 1 \\
White, Armenian & 1 \\
Asian, Pakistani & 1 \\
Black, African & \\
Educational level & 2 \\
No formal qualifications & 8 \\
School education & 2 \\
Diploma-level qualification & 3 \\
Degree-level qualification & 2 \\
Postgraduate degree & \\
\hline
\end{tabular}

Of the studies identified, all but two were conducted in the USA. The remaining two were conducted in Glasgow in the UK and Helsinki in Finland, and both seemed to suggest a more permissive view of clinical research compared with the US studies, suggesting that willingness to participate in clinical research may be influenced by political or cultural factors such as differing healthcare systems and varying attitudes towards healthcare. $^{12} 13$

Overall, the studies surveyed suggested several trends, but the majority used quantitative or mixed methods and did not explore in detail exactly what was driving participants to give the responses they did. In the light of these observations, we planned a qualitative study to attempt to explore patient perspectives in more detail.

\section{METHOD}

\section{Study design}

Semistructured interviews were conducted with inpatients on the Medical Admissions Unit and the Surgical Assessment Centre at the Northern General Hospital, Sheffield. Purposive sampling was used to identify participants with a diverse range of ages, genders, ethnicities and educational backgrounds, all of whom had recent experience of hospital admission with a medical emergency. The purposive sampling method was not designed to produce a sample representative of the proportional demographics of the population, but rather one including as diverse a range of people as possible, in the hope of capturing a maximally diverse range of opinion. Potential participants were excluded if they were under 18 years of age, lacked capacity to consent to taking part, were unable to speak conversational English or were too ill to be interviewed. Prospective written consent was gained from all participants. Interviews were carried out at the bedside, and typically lasted around $20 \mathrm{~min}$. These were audiotaped and transcribed by the interviewer, JMB, a fifth-year medical student. Interviews continued until thematic saturation was reached (the point at which new interviews could be fully coded without the creation of new, additional codes).

\section{Pilot study}

The initial interview schedule was devised by SG and used by JMB in a pilot study (carried out between September and October 2013) in which nine patients were interviewed. Following this, several prompts were added to some of the more open questions, as some participants in the pilot study struggled to comprehend what was being asked. These included a list of the factors that interviewees in the pilot study suggested would potentially affect their decision to participate in an emergency medical trial, and a list of the points covered in a patient information sheet to help interviewees decide what they would like to be told before agreeing to take part in such a trial. The final interview schedule is presented in the online supplementary appendix. Ethical approval for the updated interview schedule was granted in November 2013.

\section{Data analysis}

The transcripts were analysed using a grounded theory approach. In this research method, a theory is inductively derived through constant comparison of data. The iterative process of 'thematic analysis' allows themes to be pulled together and reworded as the interviews and the analysis unfold. The analysis proceeded according to the six-stage model of qualitative analysis laid out by Braun and Clarke in their 2006 paper 'Using thematic analysis in psychology' ${ }^{\text {14 }}$; once the researcher $(\mathrm{JMB})$ was familiar with the data, a set of initial 
codes were generated. The codes were grouped into putative themes, which were reviewed with the help of the other researchers, ADI and SG. These themes were then refined, and similar themes amalgamated until only a small number of 'primary' themes remained. These themes would form the basis of the write-up.

Owing to the time limitations of the study, it was unfortunately not possible to meet with any of the subjects again to discuss their thoughts on the final themes.

\section{RESULTS}

Twenty interviews were conducted between November 2013 and March 2014, and of these, 17 were analysed for the study. Of the remaining three, two were initiated but terminated early when the interview process revealed that the participants were too confused to give truly considered answers, and one was excluded because the responses could not be adequately discerned from the noisy recording.

The demographic details of the participants whose interviews were used in the analysis are shown in table 1 .

All interviews were carried out by the patient's bedside in the ward where they were initially approached.

The interview schedule was adhered to fairly close throughout, although with departures from the preset questions to explore any areas of particular interest that arose during the conversation. Questions were occasionally asked out of order if they seemed particularly relevant to the direction of the conversation at any given time, in the hope that they would have more meaning if viewed in the context of a participant's previous comments. The interviews selected for analysis ranged from 9 to $32 \mathrm{~min}$ in length.

\section{Themes}

The following five major themes emerged from the analysis as being central to the participants' thoughts and opinions on the idea of taking part in clinical trials within emergency care.

- Level of trust in the medical profession

- Concerns for oneself

- Altruism

- Autonomy

- Uncertainty and the importance of giving 'socially acceptable' answers

\section{Trust}

Levels of trust varied significantly between participants. A majority of the interviewees expressed themes of trust in the medical profession, and two interviewees were so trusting that they stated they would agree to any kind of trial, justifying it by expressing their certainty that the doctors would have their best interests at heart. For example, when asked about feelings on being given a trial treatment while unable to give consent, P05 answered, "At the end of the day, whatever the doctors have done, they've done for my benefit" and P04 stated "If I came in and I didn't know what was happening, whatever they give me, it's got to be for my benefit, hasn't it?"

By contrast, some participants viewed the medical profession and the concept of clinical trials with suspicion or mistrust. Some had been put off the idea of taking part in research as a result of media portrayals of clinical trials-P17 made reference to the widespread media coverage of the first-in-man trial of the immunomodulator TGN1412 at Northwick Park Hospital in 2006, which rapidly caused multiple organ failure in all six of the participants to whom it was administered. ${ }^{15}$ Discussing the patients in the trial, she said, "They were very, very ill. That's the only thing that worries me about taking part-not knowing, because you don't know what the side effects will be." Other participants mentioned previous negative experiences with healthcare or expressed frustration with their current circumstances, with the implication that these perceived inadequacies in their care would make them less open to taking part in clinical research. Probably due partly to the concurrent media controversy over the establishment of an online medical records database, two participants expressed concerns over data protection-P11 stated that she had "already opted out of NHS data being used!", while P14 stated that confidentiality has "got a bad press recently, hasn't it? With NHS records and so on..." Both went on to suggest that they thought these concerns would affect their decision to take part in a trial.

On the whole, however, levels of trust in the medical profession seemed fairly high, with only four participants expressing mistrust or suspicion.

\section{Concerns for oneself}

Many of the interviewees stated that the subjective severity of their condition during their admission would impact on their decision to take part in an emergency medical trial. Interestingly, interviewees were divided over the direction in which such a situation would push their sympathies. At least four stated that they would be less inclined to try an unproven treatment when they perceived themselves to be seriously illP03 stated

\begin{abstract}
"I suppose if you come into A\&E it depends what you come in with-I mean, I thought I could be having a heart attack, and in that case I'd have wanted what was tried \& tested. But if it was something less life-threatening, I probably would be open to a trial."
\end{abstract}

Another interviewee, P07, who at the time of interview had undergone two hospital admissions within a week, stated

\begin{abstract}
"I think emergency medicine and trials is a really tricky situation. You know, if you'd asked me about trials in the admission I'd had 3 days earlier [supraventricular tachycardia], I'd have been more open because I wasn't as unwell, and it was a more controlled situation, whereas yesterday I spent, what, 4 or 5 hours in resus [with an acute exacerbation of asthma]."
\end{abstract}

When asked whether she thought the severity of her condition would have affected her decision, she answered "Yes, definitely."

However, three participants expressed the opposite sentiment. When asked how she would feel about signing a consent form for an emergency medicine trial, P17 stated "I probably would have, without even reading it, because you do get into panic mode, don't you? Anything to help you get out of the pain. It's frightening to think what you'd do when you were in a lot of pain and discomfort." P08 stated early in his interview that his participation "[Would depend] on how ill I were, if that's an answer!" and later stated "Yeah, it's one of them where, through my own personal experiences, if you're that down, you will try anything that's going to help you." When asked if he thought being severely ill would make him more likely to take part in a trial, he answered "Yeah, it would. [...] Everybody wants to preserve life, don't they? So if someone's dangling something or if you've got something there, you probably would give it a try, or I would."

\section{Altruism}

Many patients expressed altruistic desires when considering their participation. Some were particularly emphatic about their 
willingness to undergo anything in the name of advancing scientific knowledge and helping one's fellow man, with two suggesting that they felt that it was their duty as users of the NHS. For example, P04 stated "Everything you're doing has got to benefit hospitals and it's got to come back to the patients. I'd be willing to do anything." Another patient, P09, referenced the relatively recent development of laparoscopic surgery, saying of it "I mean, and that's a massive advancement, so if I could help in something like that, absolutely." He repeatedly emphasised his belief in the importance of 'helping out', and closed the interview by saying "I'm quite a geek! I read comic books a lot, and I know I can't be a real superhero so I try and save lives like this!"

\section{Autonomy}

Several patients expressed concerns over the potential violation of autonomy posed by emergency medical trials, particularly those conducted with deferred consent. P02, when asked how she might feel on discovering that during an emergency admission she had been given a trial treatment without her consent, answered "I think I'd probably be a little bit annoyed, unless there was a family member there to discuss it with the doctors." Some had objections to the concept, but stated that their feelings on the issue would depend on the state they were in when they regained capacity. For example, when asked the same question, P20 answered "Probably aggrieved, and I'd certainly want to know the full details as to why" and later said "If I woke up, I'd probably be grateful for it! But if I woke up in a vegetative state, you know, it might be different."

\section{Uncertainty and the importance of giving 'socially acceptable' answers}

Finally, at least three interviewees seemed to be unwilling to fully commit to answers, particularly to the more complex questions. For instance, when asked how he would feel about being enrolled in research without his consent, P12 answered "Er... good question! [...] I don't know how I'd feel. If it benefited me like you said, I'd be quite happy as long as I was alright, so I don't really know on that one, to be honest." At least two interviewees seemed to seek confirmation from the interviewer when answering-P08, when asked which pieces of information he would have to be told before agreeing to take part in a trial, answered "No, erm... well basically just the dangers of what could happen, to what the, well, what were the positive side, and does it outweigh the negative side of them. Yeah?"

\section{DISCUSSION}

Overall, the results would seem to suggest that the majority of interviewees were open to the idea of taking part in research in emergency medical settings, with only one interviewee stating that she would not wish to participate at all.

The level of trust in the medical profession clearly had a big effect on patient views of willingness to participate. Some patients had such high levels of trust that they seemed entirely open to the idea of participation with no apparent caveats. There are a number of possible reasons behind this attitude of 'automatic trust'. First, it is possible that these patients simply view clinical research as so important that they are happy to undergo inconvenience and potential risk to help further the development of scientific understanding. However, it is also possible that this unquestioning openness was exaggerated by the circumstances of the interview. Given that all interviews were conducted at the patients' bedsides on a hospital ward by a medical student, it is likely that some participants were less vocally critical of the medical establishment than they would otherwise have been, possibly from a desire to give socially acceptable answers and to avoid the feeling of having caused offence. Furthermore, the concept of automatic trust tended to be expressed more frequently by the interviewees with a lower educational level, so it is possible that this attitude came about (at least in part) as a response to a perceived educational gap between interviewer and interviewee. If someone who perceived such a gap hesitated to raise objections to the idea of hypothetical research scenarios while being interviewed by a medical student, it is likely that they would have similar trouble in a real situation in which they are considering participation in an emergency medical trial. This could lead to their being enrolled against their will, or at least against their better judgement. This serves to highlight the importance for all clinicians of taking all possible steps to minimise this gap between doctor and patient. This perceived gap may also have contributed to the hesitancy some interviewees felt to fully commit to answers, or to seek confirmation of their answers from the interviewer.

When asked what they thought would affect their decision to take part in an emergency medical trial, the most common answer interviewees gave first was how ill they felt at the time. It is understandable that many would rather be given an established treatment in a situation they perceived as life threatening - after all, if taking part in clinical research is viewed as an act of self-sacrifice to help others, it is not hard to imagine that patients who would otherwise be open to the idea might be reluctant to authorise any perceived compromises to their care in a life-and-death situation. At least two of these interviewees, however, stated explicitly that their unwillingness would be due not to the unproven nature of the treatment, but to the fact that they felt that, in such a situation, they would be unable to understand the information given to them, and therefore unable to make an informed choice. This emphasis placed on the importance of understanding arose across several of the interviews, with no particular trend with educational level, gender or age. Many patients stated that they would be happy to take part in clinical research on the condition that the medical staff took the time to explain to them the purpose of the trial and what participation would involve, whether this took place before or after the treatment was administered.

The importance of understanding is further borne out by the fact that all four of the interviewees who stated that they had previous experience of clinical trials held positive views of the clinical research process. Having taken part in trials before (though not in an emergency care setting), it is reasonable to assume that these interviewees understood the process of clinical research better than most, and their positive ideas towards the process suggests that a good understanding of the process and purpose of research is a positive predictive factor for willingness to participate. Conversely, some participants seemed to struggle to fully understand the inherent element of risk involved. Some seemed to be under the impression that any clinical trial carried out on unpaid members of the public was simply a final check on top of an already convincing body of evidence, rather than the primary means by which efficacy is established. This is understandable-after all, the concept of evidence-based medicine is widely ingrained in the public consciousness, and the idea that patients might be given unproven treatments as a relatively routine aspect of a medical admission might seem strange. Overall, then, it seems that public understanding of the research process should not be taken for granted, and any strategy for maximising participant satisfaction in emergency medicine research would do well to focus on ensuring the details of the 
trial are communicated to the participant as clearly as possible. This should be considered when designing patient information sheets, since it suggests that communicating the information to the patient effectively is very important in maximising participation in clinical trials.

Some interviewees stated that they felt uncomfortable with the idea of research without prior consent because it represented a violation of patients' autonomy. There is no easy solution to this, other than to carefully explain to the patient at the earliest available opportunity exactly what was done to them and why, and to make it clear that they can elect to have their data discounted from the trial if they wish. Although difficult to alleviate, such uneasiness could be minimised through effective use of PPI when designing clinical trials, to ensure that members of the public have a forum in which to express concerns and influence the direction of research affecting them.

\section{Limitations}

Although the participants were sampled from a group of hospital inpatients with recent experience of an emergency medical admission, none of them had prior experience of an emergency medical trial, and as a result it is possible that the answers they gave are not truly reflective of how they would react in a real emergency scenario. In addition, it is possible that the interviewer's status as a medical student had an effect on the answers that participants gave. Although purposive sampling achieved a diverse mix of ages, genders and educational levels among the interviewees, the largely White British makeup of the local area, coupled with the limited timeframe of the study, meant that the sample did not span as many different ethnicities as would have been preferable. The sample was also limited to people who could speak conversational English, and although no participants were actually excluded from the study on this basis, it is possible that such a group may have different views to express. It is also possible that, since the study was limited to those who consented to take part, a potential demographic has been missed in those who did not agree to do so, even though only two people declined to take part in the study, there is a chance that those who are not willing to participate in a study like this would hold differing views on clinical research to those who are. Finally, it should be stated that the aim of this study was not to draw generalisable conclusions from a representative sample, but to use a maximally diverse sample to identify all the significant concerns and feelings that would be held by a diverse population.

\section{Conclusions}

Among the 17 patients interviewed, attitudes towards clinical research in emergency care were largely positive. Factors identified as affecting interviewees' willingness to participate in a hypothetical emergency medical trial included level of trust in the medical profession, concerns for oneself, altruistic tendencies and concerns over autonomy. Many interviewees stressed the importance they would place on being kept as well-informed as possible at every stage of the process.

\section{Recommendations}

Given the earlier discussion of the importance to participants of being well-informed, maximising patient acceptance of clinical research requires the provision of accurate and understandable information for participants at every stage of the research process. In addition to consultations with PPI groups and raising public awareness (eg, by more actively publicising research in the mainstream media), this could potentially be achieved by being more creative with the means by which the information is communicated to potential trial participants. When asked, most interviewees stated they would not have a problem with an abridged patient information sheet, so long as they received the remaining details at a later date. If we agree that this is acceptable in principle, many other ways of communicating the essential points to the patient could be exploredfor example, the information could be presented in graphical form, in the manner of a decision aid or flow chart, or even a video presented to the patient on a tablet computer. Presented in this manner, the information is likely to be easier for a distressed patient to truly absorb.

Funding $f 1000$ personal BMedSci funding received from the University of Sheffield. Competing interests None declared.

Ethics approval National Research Ethics Committee, Leicester Branch.

Provenance and peer review Not commissioned; externally peer reviewed.

Data sharing statement The full dissertation upon which this paper is based is available from the author on request.

\section{REFERENCES}

1 Roberts I, Prieto-Merino D, Shakur $\mathrm{H}$, et al. Effect of consent rituals on mortality in emergency care research. Lancet 2011;377:1071-2.

2 Trial m. 3mg Trial Protocol. 2010. https://www.shef.ac.uk/polopoly_fs/1.44177!/file/ 3Mg-Protocol-v006-10AUG2010.pdf

3 (IST-3) IST. International Stroke Trial 3 Protocol. 2009. http://www.den.ed.ac.uk/ist3/ Web_docs_110310/Protocol\%20\&\%20Summary/IST3\%20Protocol\%20Version\% 201_93\%2030th\%20March\%2009_7_signed\%20copy.pdf

4 CRASH2. CRASH2 Trial-Procedure for Obtaining Consent (EU). 2005. http://www. crash2.Ishtm.ac.uk/prot_EngConsentProcedure.htm

5 US Department of Health and Human Services. Informed Consent Requirements in Emergency Research. 1996. http://www.hhs.gov/ohrp/policy/hsdc97-01.html

6 Lecouturier J, Rodgers H, Ford GA, et al. Clinical research without consent in adults in the emergency setting: a review of patient and public views. BMC Med Ethics 2008;9:9.

7 Nelson MJ, Delorio NM, Schmidt T, et al. Local media influence on opting out from an exception from informed consent trial. Ann Emerg Med 2010;55:1-8.

8 Nelson MJ, Deiorio NM, Schmidt TA, et al. Why persons choose to opt out of an exception from informed consent cardiac arrest trial. Resuscitation 2013;84: 825-30.

9 Biros MH, Sargent C, Miller K. Community attitudes towards emergency research and exception from informed consent. Resuscitation 2009;80:1382-7.

10 Dickert NW, Mah VA, Baren JM, et al. Enrollment in research under exception from informed consent: the Patients' Experiences in Emergency Research (PEER) study. Resuscitation 2013;84:1416-21.

11 Dickert NW, Kass NE. Patients' perceptions of research in emergency settings: a study of survivors of sudden cardiac death. Soc Sci Med 2009;68:183-91.

12 Kämäräinen $A$, Silfvast $T$, Saarinen $S$, et al. Conduct of emergency research in patients unable to give consent-Experiences and perceptions of patients, their consent providing next of kin, and treating physicians following a prehospital resuscitation trial. Resuscitation 2012;83:81-5.

13 Booth $M$, Lind $A$, Read $E$, et al. Public perception of emergency research: a questionnaire. Eur J Anaesthesiol 2005;22:933-7.

14 Braun V, Clarke V. Using thematic analysis in psychology. Qual Res Psychol 2006;3:77-101.

15 Vince G. UK drug trial disaster-the official report. 2006. http://www.newscientist. com/article/dn9226-uk-drug-trial-disaster--the-official-report.htm|\#.U3Awi_IdUic 